\title{
Demonstrating the Architecture for Situation-aware Logistics using Smart Returnable Assets
}

\author{
Jean Paul Sebastian Piest \\ University of Twente \\ Enschede, The Netherlands \\ j.p.s.piest@utwente.nl \\ https://orcid.org/0000-0002-0995-6813
}

\author{
Rob Henk Bemthuis \\ University of Twente \\ Enschede, The Netherlands \\ r.h.bemthuis@utwente.nl \\ https://orcid.org/0000-0003-2791-6070
}

\author{
Gilang Charismadiptya \\ CAPE Groep \\ Enschede, The Netherlands \\ g.charismadiptya@capegroep.nl \\ https://orcid.org/0000-0002-7694-4759
}

\begin{abstract}
Building on earlier work, this paper aims to demonstrate and discuss an instantiated architecture for situation-aware logistics in an operational environment using smart returnable assets. The demonstration is based on a motivation scenario focusing on exception management. The system outline and its components, interfaces, and enabling technologies are described and linked to the different layers of the architecture. This paper documents and illustrates the use of the system with detailed models and screenshots. Earlier work is extended using business rules to identify and quantify exceptions and potential disruptions. Specifically, it is shown how shipment data are enhanced with data from IoT sensors of smart returnable assets to provide situation-aware decision support based on data analytical methods. This demonstration provides scholars and practitioners, active in the fields of enterprise computing, insights into the concepts, models and engineering technologies used to implement an architecture for situation-aware logistics. The instantiated architecture provides a rich testbed for experiments, measurements, and incorporate the ideas promoted by the international data space initiative. An online recording is available to support the demonstration and ignite discussion about the potential of IoT technologies and future research directions in pursuit of the smart logistics vision.
\end{abstract}

Keywords—architecture, iot, smart logistics, business rules, exception management

\section{INTRODUCTION}

The smart logistics vision constitutes digitally interconnecting smart assets, applications, components, devices, and users within both an intra- and inter-enterprise context. This vision is not just about connecting with one site of an enterprise, but about leveraging cloud and internet opportunities by connecting multiple sites to form a multitude of digital supply chains [1]. Although contemporary efforts in the smart logistics and supply chain domain are staggering, studies report that the current influence of IoT applications in supply chains is still at a preliminary stage. Further steps need to be undertaken to show its full technological potential $[1,2]$.

In this paper, we focus on the management of exceptions in a smart logistics context. Exception handling is an important capability that contributes to the building of resilient enterprises. An exception is any phenomenon that prevents the successful completion of normal business processes [3]. We emphasize the interfacing role of business rules with resilience in mind, which can help in understanding, managing, and improving logistics processes [4]. A business rule is a statement that aims to influence or guide behavior and information in an organization [5]. Resilience is often referred to as "the capability of an element to return to a predisturbance, stable state after a disruption" [6]. In the context of this research, resilience is defined as "the ability to sense, recognize, adapt and absorb variations, changes, disturbances, disruptions and surprises" [7]. The underlying research focuses on devising a new way of anticipating disruptions arising from Smart Returnable Assets (SRAs) and its contribution to building resilient enterprises. GS1 defines a returnable asset as "a reusable package or transport equipment of a certain value" [8]. The Global Returnable Asset Identifier (GRAI) enables uniform identification of returnable assets, individual tracking and recording of data [8]. In the context of this research, an SRA is defined as a reusable package or transport equipment that is employed with IoT technology and digitally interconnected to a multitude of supply chains. In this paper, we evaluate the use of smart pallets that are deployed in a closed loop circuit to gather situational data for exception management, business rule enactment, and decision support.

This paper mainly builds on the Situation-aware Smart Logistics Enterprise Architecture (SSLEA), as presented in [9], aiming to demonstrate and discuss its use in an operational environment using SRAs. Although the proposed architecture is based on key principles from an extensive requirement analysis, literature review, and ideas promoted by the International Data Space (IDS) initiative [10], the level of implementation and evaluation is limited to validation using a prototype. In this paper, we present the results of our efforts to move towards implementation in an operational environment using a motivation scenario focusing on realtime management of exceptions in pursuit of the smart logistics vision. More specifically, the prototype of [9] is further developed into an application and integrated with a cloud environment of the technology provider of SRAs via APIs. Additionally, we show how IoT-based event monitoring can be used to assist decision-makers by illustrating the use and functioning of a hierarchical decision support system based on business rules.

This paper is structured as follows. Section II provides a system outline and describes the components, interfaces, and enabling technologies. Section III describes the motivation scenario that is used to demonstrate the system in an operational logistics context. Section IV discusses the results, concludes, and provides an outlook on future work.

\section{SyStem OUTLINE}

This section first describes the system overview, referring to the SSLEA. Subsequently, the main system components and interfaces are described.

\section{A. Overview}

Based on the SSLEA as presented in the paper [9], a distributed system is designed and developed. This system supports exception management in an operational environment using SRAs without intervening in the primary logistics processes. Figure 1 depicts the system overview. In the business layer, processes related to exception management are realized and supported by a situation-aware logistics service according to the SSLEA. The application layer contains a Shipment Scheduling Application (SSA), Situation-aware Logistics Information System (SLIS), and IoT Cloud Application (ICA). The technology layer consists 


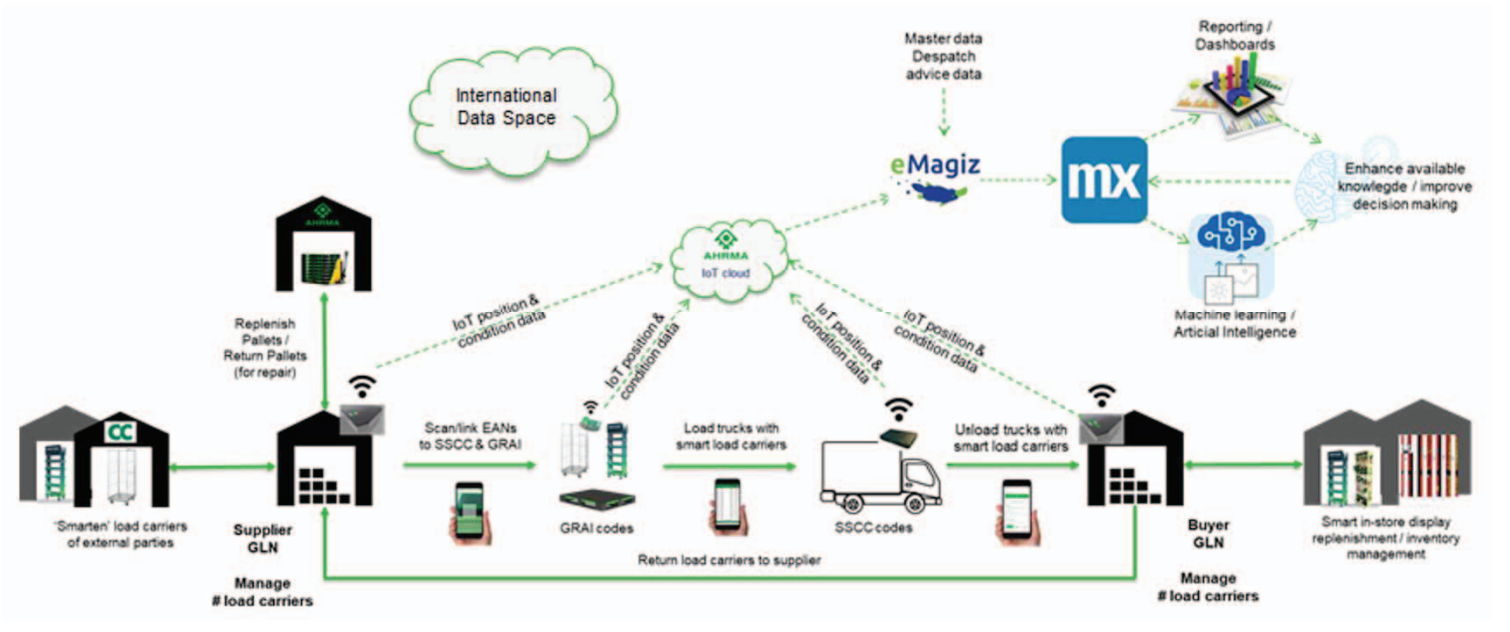

Figure 1. System overview and motivation scenario.

of application servers and databases to support the information systems, an integration platform that provisions connectors, pipelines and message queues, and a physical pool of SRAs with corresponding computation nodes. Given the current state of development and implementation of the IDS initiative, the ideas, concepts, and technologies related to IDS will be incorporated in a future design and development cycle.

\section{B. Components}

The situation-aware logistics service, as described in the SLLEA, is realized by the SSA and SLIS, which both provide a web-based user interface and web services. The SRAs are administered and assigned to physical locations and users in the ICA. Shipment data are administered in the SSA. Customer and order data, originating from the SSA or an ERP system, and IoT data from the ICA are aggregated in the SLIS. This way, rather static data are enriched with real-time sensor data originating from one or multiple pools of SRAs. Based on configured business rules and thresholds, exception logic is created and stored in the knowledge base of the SLIS. Exceptions are associated with shipments and corresponding order lines. Based on user preferences, stakeholders are notified about the shipment status and exceptions.

\section{Interfaces}

The SLIS is integrated with the SSA and the ICA of an SRAs provider via APIs. Data exchange and semantic transformations are realized through an integration platform, which also provides a message queuing service. The ICA is connected to a physical pool of SRAs via gateways in, for example, warehouses, trailers or, mobile devices. Raw sensor data measurements are (temporary) stored on edge/fog-based IT infrastructures.

\section{DEMONSTRATION}

This section describes the motivation scenario that is used to demonstrate and discuss how the SSLEA can be implemented and used in an operational context using SRAs.

\section{A. Motivation scenario}

Figure 1 depicts the motivation scenario that is used for the demonstration of the SSLEA. The scenario is instantiated

\footnotetext{
${ }^{1}$ http://ahrmagroup.com/

2 https://www.emagiz.com/
}

using the SRAs and IoT technologies of Ahrma ${ }^{1}$, the integration platform as a service of $\mathrm{eMagiz}^{2}$, and application platform as a service of Mendix ${ }^{3}$. The scenario of SRAs is chosen for the following reasons:

1. The requirements of the stakeholders and users can be satisfied. For example, the motivation viewpoint of the transportation manager, described in [9], can be realized and evaluated.

2. IoT assets are linked to shipments and deployed in a closed circuit and should, eventually, be returned. This requires active track and trace functionality.

3. The system can be connected to the operational pallet pools without intervening in the operational logistics process and without disclosing privacy-sensitive data.

4. Different types of pallet pools can be monitored and compared, providing a rich testbed for system development and experimentation. This testbed can be valuable for assessing strategies for resilience, knowledge enhancements, and decision support.

5. Business rules and thresholds can be deployed on different levels (e.g., edge, fog, cloud) and for different types of IoT data.

6. Access is provided to historical data and real-time data, making it, for example, possible to implement and compare different data mining and machine learning approaches, apply operations research techniques for problem solving and optimization, and algorithms for process mining and event streaming.

7. The scenario is supported by a distributed system, covering both intra- and interorganizational processes and involving multiple stakeholders, thus, providing a realistic environment for validation.

8. The set-up allows including assets and load carriers of third parties and extension of use cases such as condition monitoring, product recall, in-store display inventory monitoring, and replenishment. 
9. The systems are compatible with industrial standards (e.g., GS1 barcodes, ID keys, data sharing models).

10. The scenario can be extended to support data sharing initiatives, such as propagated by IDS. This way, it can also function as a testbed for the development of IDS connectors and use cases for secure data sharing.

11. Next to economic value and efficiency, using SRAs contributes to sustainability and life-cycle extension.

\section{B. Smart returnable assets and gateways}

The smart pallets [11] of Ahrma, depicted in Figure 2, are used as the SRA, and include transponder technology to capture and share real-time measurements of its location, movements, shocks, temperature, weight, and humidity.
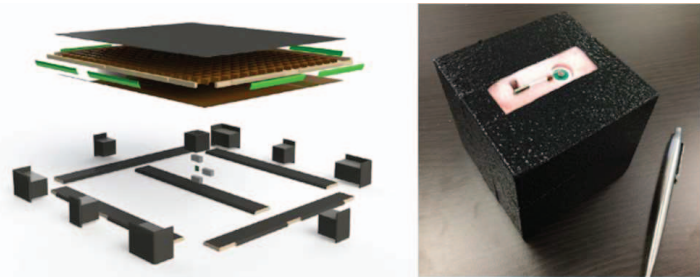

Figure 2. Smart pallet and transponder technology, images [11].

Different gateway solutions are used to build an IoT network and send collected measurements to the ICA Stationary gateways can be used indoor, e.g., in a warehouse or production facility. Mobile gateways can be attached to equipment, e.g. in a trailer. In addition, a smartphone can be used as a mobile gateway in the field via the Ahrma app.

\section{IoT cloud application, dashboard, and API}

The ICA provides a dashboard with (1) a real-time map, (2) data analytics and statistics (e.g. locations, pool inventory, movements, stationary), (3) asset control charts (e.g., temperature, humidity, shocks, and weight), (4) asset lifecycle management charts (e.g., battery level, signal strength), (5) configuration of events, alerts, and notifications, and (6) management of assets, gateways, sites, business partners and users. The main features are illustrated in Figure 3, 4, and 5.

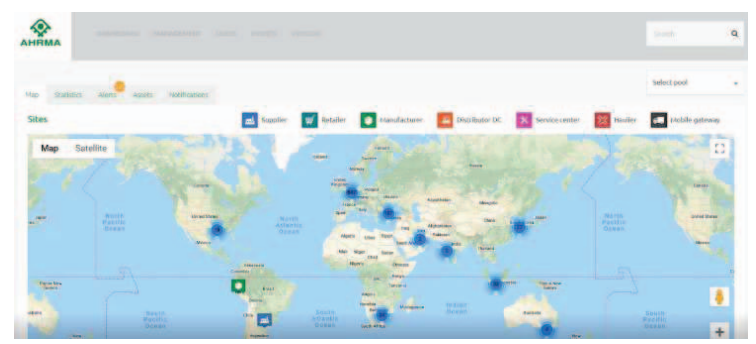

Figure 3. Real-time map displaying multiple SRA pools.

Ahrma provides third parties shipment and asset tracking information via an API. Authentication and authorization are based on the OAuth 2.0 protocol. The API uses the web socket protocol. Messages are sent and received using the STOMP messaging protocol. Request and response messages are formatted in JSON. For this demonstration, the shipment endpoint is used to create a subscription for shipments, and receive updates of the location, movements, and related data measurements of the associated SRAs.

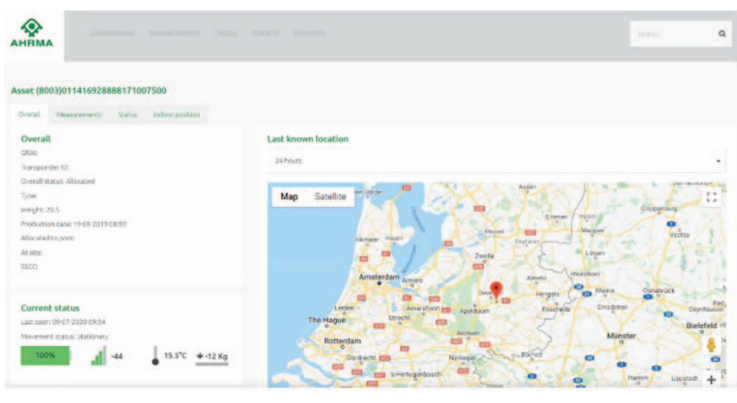

Figure 4. Real-time SRA monitoring and measurements.

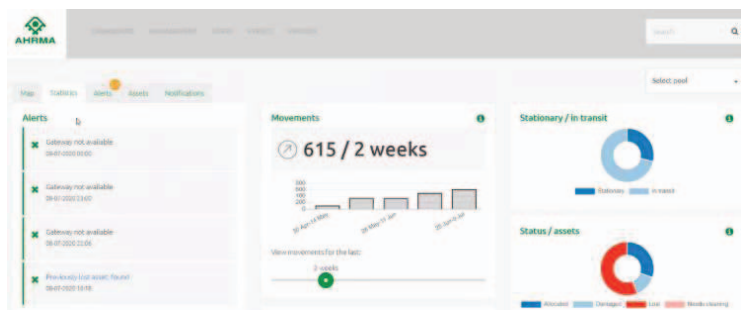

Figure 5. Data analytics and statistics.

The shipment API provides a data stream for third parties to track their shipments and share detailed information with customers about the current location and status. A shipment consists of one or multiple SRAs that can be tracked together. SRAs are registered in a shipment by their GRAI code and usage standards. This GS1 code (the barcode) is displayed on every SRA. A shipment can be created by submitting one or more GRAI codes to the shipment endpoint. Active shipments can be updated by submitting one or more GRAI codes to the shipment endpoint. The previous barcodes will be replaced by the newly submitted barcodes. Shipments can be also be canceled or deleted. Thus, the API supports the full lifecycle.

After connecting to the API, a subscription can be taken on the level of a shipment or individual SRA. Data updates are pushed through the web socket connection at a regular interval. Upon arrival or when a shipment is canceled, the latest status will be sent over the subscription and will be closed afterward. The shipment status is determined by the location of the SRA. When the SRAs leave the departure location, the shipment status will change from "awaiting" to "in transit". Upon arrival at the destination location, the shipment status will be set to "completed".

\section{Integration platform}

The eMagiz integration platform realizes connections with the web services of the SSA, the Ahrma API, and the SLIS. Subsequently, event data are streamed based on subscriptions, transformed, and updated in the SLIS. Figure 6 depicts the systems (blue objects) and interfaces (green lines).

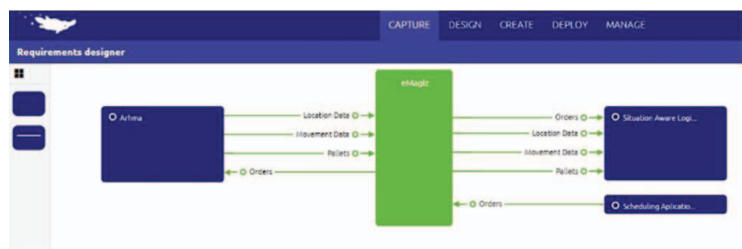

Fig 6. Overview of systems and interfaces in eMagiz. 


\section{E. Situation-aware logistics information system}

The Mendix application platform is used to develop the SLIS according to the SSLEA. The SLIS provides a (1) dashboard, (2) notifications, (3) shipment administration, (4) pallet administration, (5) reports, (6) master data management, and (7) user and system administration. Figure 7 depicts the dashboard. Shipment and IoT data are received from the SSA and ICA via SOAP XML web services.

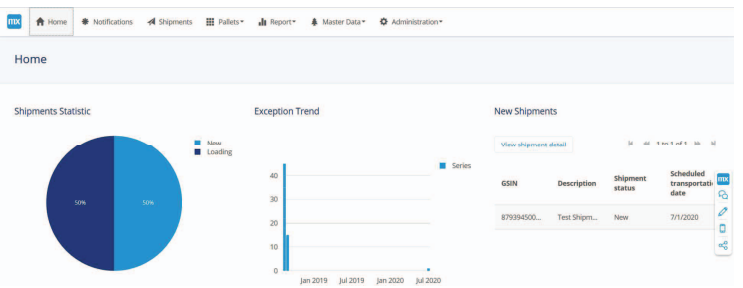

Figure 7. Situation-aware logistics information system.

\section{F. Shipment scheduling application}

The SSA is also developed using the Mendix application platform. The SSA, as shown in Figure 8, provides functionality for (1) master data management and (2) shipment administration. Master data includes locations, vehicles, and drivers. Shipment data includes stakeholders, locations, goods, SRAs, and details. Figure 8 depicts the shipment overview, which allows users to create shipments. Shipments data are shared using SOAP XML web services.

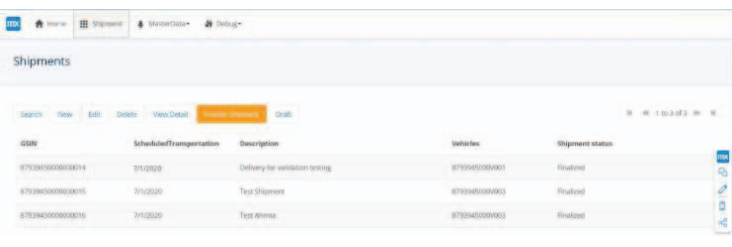

Figure 8. Shipment scheduling application.

\section{$G$. Business logic and rules}

The ICA of Ahrma supports the use of business rules, which are executed in real-time or in batch. Alerts are created if an SRA, for example, arrived at a site, is marked as damaged and/or lost, has entered a different pool, or when a lost asset is allocated again. Thresholds can be set to measure if the asset temperature, shock, and/or weight is within, above or below allowed values. Furthermore, alerts are created if a gateway goes down unexpectedly or if an update failed or succeeded. Together with battery level and signal strength, these values can be used for lifecycle management and predictive maintenance. In the current and future work, as outlined in $[4,12]$, we plan to employ further analytics methods by using business rules, process mining, and agent-based modeling.

\section{H. Exception rules and management}

The SLIS allows users to create exception rules. These rules are linked to item categories to inspect incoming sensor data. Figure 9 illustrates how users can configure exception rules based on temperature data. Currently, exception rules can be configured for temperature, humidity, shock, and location. The SLIS utilizes configured exception priorities to generate notifications, warnings or raise immediate attention to the user. Raw sensor data can produce large amounts of event data. Therefore, an aggregation function is created to prevent users to be overwhelmed by exception notifications.

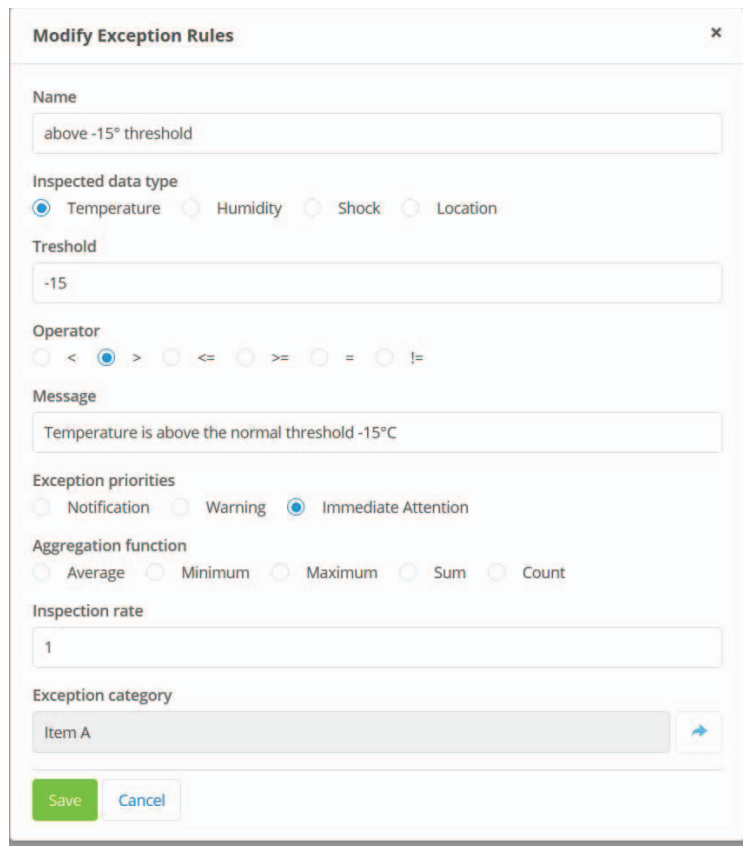

Figure 9. Configuration of exception rules.

\section{Process mining and event streaming algorithms}

Inspired from the work of [12], the authors are currently developing a series of process mining, event streaming, and pattern learning algorithms, which can be incorporated into decision-support systems. Process mining techniques will be applied to mine the event logs of transponders, and aggregate movement and location data. Historical data are available up to one year and provide a rich testbed to develop, test and compare different algorithms. Real-time streaming event data are used to identify, assess, and (if possible) resolve logistical disturbances and develop dynamic optimization algorithms as proposed by [13]. In addition, the input and involvement of the users will be incorporated using the principles of intelligent amplification, as proposed by $[13,14]$.

\section{DisCUSSION, CONCLUSION AND OUTLOOK}

This section summarizes and discusses the results and findings, concludes the work, and presents an outlook.

\section{A. Summary of results and findings}

This paper demonstrates how the SSLEA can be implemented using platforms and technologies related to SRAs. The functioning of the system is demonstrated based on a motivation scenario, facilitating the enactment of IoTdriven event streams, business rules, and data analytics. The system has been instantiated, tested, and configured to monitor operational logistics processes related to the motivation scenario, and manage exceptions based on business rules and real-time data originating from pools of SRAs with actual movements. Shipment data are enriched with IoT data originating from an operational pool of pallets using the transponders, gateways, and IoT network. Both static and real-time data are made accessible via APIs and exchanged via an integration platform. In addition to this paper, the use of the system is captured in a screencast available at https://youtu.be/qiN_eB7Q-Xk. 


\section{B. Discussion}

This paper presents efforts in implementing and extending the work as presented in [9]. Particularly, we focused on validation by means of a real-life case study implementation of the SSLEA. The exception handling process, situationaware logistics service, corresponding application functions, and supporting data and processing services, described in [9], are mostly realized. This demonstration provides empirical support and insights to further develop, validate, and evaluate the SSLEA. The demonstration incorporates multiple topics in the field of enterprise computing, e.g., enterprise architecture, business intelligence and business analytics, and business rules. Both researchers and practitioners can see validations of earlier work and real deployments in the context of distributed enterprise applications and systems, originating from crossdisciplinary research projects of $[4,13,14]$. The demonstration is considered of interest to both researchers and practitioners active in the field of enterprise computing.

\section{Conclusion}

In this paper, instantiation of the SSLEA is demonstrated and discussed in an operational environment using SRAs. Earlier work is enhanced by considering various sources of disruptions, strategies of negating disruptions in the form of business rules, and the use of data analytical techniques. The demonstration contributes to exploration and exploitation of the potential of the smart logistics vision and provides a rich testbed for further development and experiments. More specifically, the motivation scenario and instantiated SSLEA for SRAs provide a realistic environment to assess the use, value, implications, and impact of IoT data in relation to exception management and operational decision making. In addition, collaboration with industry and compliance with industrial GS1 standards contributes to consolidation in the industry. However, the implementation of the SSLEA for SRAs has its limitations. The activities related to exception management, gathering of information, and notifying customers are realized and supported by application services. Generating, executing, and evaluating a mitigation plan is not yet supported by an application service and requires further empirical research and system development. Given the current state of development of the IDS, the information sharing process, described in [9] could not be realized. Furthermore, the instantiation of the SSLEA is limited to SRAs and should be tested in multiple IoT use cases and environments using different configurations and alternative technologies.

\section{Future work}

In future work, the remaining parts of the SSLEA for SRAs will be realized, tested for scalability and robustness, and its impact will be assessed by analyzing and comparing multiple pools of SRAs. More specifically, different business rule configurations, and techniques for data analytics, process mining, and operations research will be tested and evaluated using both historical and real-time data. Furthermore, interoperability with IDS [10] will be examined, as well as the integration with sophisticated analytical decision support systems. Another promising direction is the incorporation of an intelligence amplification approach, as positioned in [14]. Such an extension would be useful for determining which parts of the system can be taken over by intelligent agents, which parts humans may need to intervene with the system and how symbiosis supports operational decision making and contributes to the overall system performance.

\section{ACKNOWLEDGMENT}

This research is financially supported by the Dutch Ministry of Economic Affairs and co-financed via TKI DINALOG and NWO. Funding for this work has been granted by the DataRel project (grant no. 628.009.015), the ICCOS project (grant no. 2018-2-169TKI) and CLICKS project (grant no. 439.19.633). The authors thank Martijn Koot and Maria Eugenia Iacob for the fruitful discussion about the motivation scenario and the anonymous reviewers for their constructive feedback. A special word of thanks is for Bart Knol of CAPE Groep and Erik Ekkel of Ahrma for making the technology and resources available for conducting this research.

\section{REFERENCES}

[1] E. Manavalan and K. Jayakrishna, "A review of internet of things (iot) embedded sustainable supply chain for industry 4.0 requirements,"Computers \& Industrial Engineering, vol. 127, pp. 925953, 2019. https://doi.org/10.1016/j.cie.2018.11.030.

[2] M. Ben-Daya, E. Hassini, and Z. Bahroun, "Internet of things and supply chain management: a literature review,'International Journal of Production Research, vol. 57, no. 15-16, pp. 4719-4742, 2019 https://doi.org/10.1080/00207543.2017.1402140.

[3] M. Klein, C. Dellarocas, and A. Bernstein, "Introduction to the special issue on adaptive workflow systems," Computer Supported Cooperative Work, vol. 9, no. 3-4, pp. 265-267, 2000. https://doi.org/10.1023/A:1008764508237.

[4] R. Bemthuis, "Business logic for resilient supply chain logistics," in 2019 IEEE 23rd International Enterprise Distributed Object Computing Workshop (EDOCW). IEEE, 2019, pp. 190-195. https://doi.org/10.1109/EDOCW.2019.00041.

[5] G. Steinke and C. Nickolette, "Business rules as the basis of an organization's information systems, "Industrial Management \& Data Systems, 2003. https://doi.org/10.1108/02635570310456904.

[6] R. Bhamra, S. Dani \& K. Burnard, "Resilience: the concept, a literature review and future directions," International Journal of Production Research, $\quad 49: 18, \quad 5375-5393 . \quad 2011$ https://doi.org/10.1080/00207543.2011.563826.

[7] E. Hollnagel, D.D. Woods, and N. Leveson. "Resilience engineering: concepts and precepts". Aldershot: Ashgate. 2006.

[8] "GS1 General Specifications", 2015. Section 2.3.1. Global Returnable Asset Identifier (GRAI), accessed online: 6 September 2020. [Online]. Available: https://www.gs1.nl/sites/default/files/GS1 General Specifications 0. pdf

[9] M.-E. Iacob, G. Charismadiptya, M. van Sinderen, and J. P. S. Piest, "An architecture for situation-aware smart logistics," in 2019 IEEE 23rd International Enterprise Distributed Object Computing Workshop (EDOCW). IEEE, 2019, pp. 108-117. https://doi.org/10.1109/EDOCW.2019.00030

[10] "IDS Reference Architecture Model 3.0", 2019, last accessed online: 13 September 2020. [Online]. Available: https://www.internationaldataspaces.org/publications/referencearchitecture-model-3-0/

[11] "Ahrma infograpgic", 2020, last accessed online: 13 September 2020 [Online]. http://ahrmagroup.com/downloads/ahrma infographic.pdf

[12] R. H. Bemthuis, M. Koot, M. R. Mes, F. A. Bukhsh, M.-E. Iacob, and N. Meratnia, "An agent-based process mining architecture for emergent behavior analysis," in 2019 IEEE 23rd International Enterprise Distributed Object Computing Workshop (EDOCW). IEEE, 2019,pp.54-64. https://doi.org/10.1109/EDOCW.2019.00022

[13] M. Koot, "Towards a framework for smart resilient logistics," in 2019IEEE 23rd International Enterprise Distributed Object Computing Workshop (EDOCW). IEEE, 2019, pp. 202-207. https://doi.org/10.1109/EDOCW.2019.00043

[14] J.P.S. Piest, "A platform architecture for industry 4.0 driven intelligence amplification in logistics," in 2019 IEEE 23rd International Enterprise Distributed Object Computing Workshop (EDOCW). IEEE, 2019, pp.174-178. https://doi.org/10.1109/EDOCW.2019.00038 